\title{
Reflexões sobre as mudanças no modelo de financiamento federal da Atenção Básica à Saúde no Brasil
}

\author{
Reflections on changes in the federal funding model of Primary \\ Health Care in Brazil
}

Eduardo Alves Melo', Patty Fidelis de Almeida², Luciana Dias de Lima', Ligia Giovanella'

DOI: 10.1590/0103-11042019S512

APÓS 25 ANOS DE IMPLANTAC̣ÃO, SÃO INCONTESTÁVEIS OS AVANC̣OS decorrentes do fortalecimento da Estratégia Saúde da Família (ESF) no Brasi11-6, em que pese a existência de limites e desafios importantes. A ESF é o principal modelo de Atenção Primária à Saúde (APS), também nominada Atenção Básica (AB), e incorpora os atributos essenciais e derivados estabelecidos por Starfield7 7 e outras formulações desenvolvidas no campo da saúde coletiva do País. Caracterizase pela conformação de equipes multiprofissionais e opera nas dimensões individual, familiar e coletiva/territorial do processo saúde saúde-doença, por meio de ações clínicas e sanitárias e estratégias de participação social ${ }^{8,9}$. Em 2019, existiam mais de 43 mil Equipes de Saúde da Família implantadas em todo o território nacional, contemplando grande diversidade de municípios e grupos populacionais ${ }^{10}$.

A implantação da ESF ocorreu de forma progressiva, envolvendo amplo debate entre diversos atores do Sistema Único de Saúde (SUS), das instituições acadêmicas e da sociedade, nos processos de formulação de políticas, como, por exemplo, a primeira Política Nacional de Atenção Básica, lançada no ano de 2006 ${ }^{11}$. As negociações buscaram equacionar diferentes pontos de vista, interesses e conflitos para a construção de consensos possíveis em cada momento histórico, respeitando o marco legal do SUS que prevê a gestão tripartite do sistema, mediada pelas instâncias de controle social12. Esse processo foi fundamental para a adequação das regras institucionais à diversidade do País, de forma coerente com os princípios de universalidade, integralidade e equidade.

No cenário atual, observa-se certa reversão da lógica de negociação das políticas públicas, com desvalorização dos mecanismos de participação e controle social na saúde, como no recente

'Fundação Oswaldo Cruz (Fiocruz), Escola Nacional de Saúde Pública Sergio Arouca (Ensp) - Rio de Janeiro (RJ), Brasil. eduardo.melo@ensp. fiocruz.br

2 Universidade Federal Fluminense (UFF), Instituto de Saúde Coletiva (ISC) Niterói (RJ), Brasil. caso da proposição do conselho consultivo da Agência de Desenvolvimento da APS (Adaps) ${ }^{\mathbf{1 3}}$, e pelo açodado processo de aprovação de um conjunto de reformas estruturantes, entre as quais, a da Previdência e a Trabalhista. Tal processo tem exigido a rápida reconfiguração do campo de forças e atores implicados na defesa de um "projeto civilizatório de inclusão e solidariedade" representado pelo SUS ${ }^{14}$.

Nesse contexto, considera-se relevante discutir as mudanças do financiamento federal da $\mathrm{AB}$ que, associado a outras propostas de reforma recentes do Ministério da Saúde, possui caráter estrutural e afeta profundamente o modelo da ESF e do próprio SUS. Cabe ressaltar que a finalização deste artigo se deu imediatamente após a aprovação pelos representantes 
das três esferas de gestão do SUS, em reunião da Comissão Intergestores Tripartite (CIT), ocorrida em 31 de outubro de 2019 , e subsequente publicação da portaria que institui novo modelo de financiamento de custeio da APS ${ }^{15}$. Nesse sentido, o objetivo deste artigo é destacar os principais elementos de mudança do modelo proposto e analisar suas possíveis implicações para a APS no Brasil.

\section{Os principais pontos de mudança na proposta do Ministério da Saúde para o financiamento da APS}

O novo modelo de financiamento federal da APS foi apresentado inicialmente no Congresso Brasileiro de Medicina de Família e Comunidade em julho de 2019, sendo divulgado de forma diversa pelos dirigentes federais em eventos com gestores (apresentações em Power Point ${ }^{\circledR}$ ), e em parte publicado em artigo de opinião em uma revista científica ${ }^{16}$. A proposta, recém-divulgada por meio de minuta de portaria federal indica mudanças significativas no padrão de financiamento instituído gradativamente no Brasil a partir da segunda metade dos anos 1990, sem, no entanto, estar acompanhada de garantia de aumento efetivo e sustentável no aporte de recursos federais para a saúde.

As justificativas para as mudanças se assentam em suposta ineficiência dos gastos em $\mathrm{AB}$, e se apoiam em experiências internacionais para realizar uma série de comparações. A mudança prevê o fim do Piso de Atenção Básica (PAB) Fixo (transferência federal per capita para todos os municípios considerando suas populações estimadas e características socioeconômicas) e da dimensão do PAB Variável relativa à implantação de Equipes de Saúde da Família (que gera valores de repasse mensais segundo tipos e números de equipes implantadas). Além disso, modifica a dimensão do PAB Variável relativa ao desempenho. Defende que a maior parte do financiamento federal se dê com base em um componente de capitação relativo à população cadastrada em Equipes de Saúde da Família e APS no Sistema de Informação em Saúde para a Atenção Básica (Sisab), e no pagamento por desempenho considerando o resultado de indicadores pelas equipes credenciadas e cadastradas no Sistema de Cadastro Nacional de Estabelecimentos de Saúde (SCNES). Prevê a manutenção de algumas equipes e programas (como saúde bucal, consultório na rua, informatização, entre outros), mas acaba com o financiamento federal dos Núcleos Ampliados de Saúde da Família e Atenção Básica (Nasf-AB).

Embora sinalize ponderação do financiamento com base em critérios de vulnerabilidade socioeconômica, demográficos e classificação geográfica de municípios, apresenta metas de cadastro (posteriormente renomeadas como potencial de cadastro) de 4 mil pessoas por equipe em municípios urbanos, não compatíveis com a prestação do cuidado integral, com base comunitária, previstas pela ESF. Aliás, não menos importante é destacar que a proposta menciona que a população deve ser cadastrada por 'Equipe de Saúde da Família e atenção primária', o que permitiria inferir não haver diferença entre o cuidado prestado pelas duas modalidades, cujos parâmetros populacionais para cobertura são os mesmos.

Outro ponto fundamental é a substituição da dimensão do PAB Variável relativa ao desempenho, representada pelo Programa de Melhoria do Acesso e da Qualidade da Atenção Básica (PMAQ-AB) desde 2011, por um conjunto de indicadores categorizados como de processo e resultados intermediários das equipes, resultados em saúde e indicadores 'globais' em APS. Ressalta-se que não há indicação sobre a forma de cálculo das metas a serem alcançadas pelas equipes, e de que modo seriam contempladas as imensas diversidades que caracterizam nosso território.

O novo modelo contém, ainda, um componente com incentivos para ações estratégicas, com subcomponentes como o Programa Saúde na Hora, Informatização e Formação 
em Residência Médica e Multiprofissional; Saúde Bucal; Agentes Comunitários de Saúde; Promoção da Saúde (Saúde na escola e Academia da Saúde); além de especificidades como Consultório na Rua, Unidades Básicas de Saúde (UBS) Fluviais, entre outros, reconhecidamente importantes na trajetória de fortalecimento da APS no País. Porém, exclui financiamento específico para as equipes de Nasf. A exclusão dos incentivos voltados para os Nasf parece incidir também sobre o caráter multiprofissional da ESF, que tinha nesses profissionais a complementariedade de saberes e práticas, a partir do apoio matricial e atuação clínica compartilhada, visando ampliar a resolutividade e capacidade de cuidado da APS.

\section{O cenário adverso ao SUS, os limites e os riscos da nova proposta de financiamento}

A proposta surge em um cenário de crise e de reformas nacionais com notável impacto sobre políticas sociais, incluindo repercussões gravíssimas sobre a saúde da população, agravadas pelas medidas de austeridade fiscal ${ }^{17}$. O SUS, desde o início de sua implementação, sofre problemas crônicos de financiamento, que foram ampliados nos últimos anos diante da intensificação de reformas calcadas na austeridade fiscal e na restrição aos gastos públicos. Ao longo de três décadas, os municípios expandiram de forma expressiva sua participação no financiamento do sistema, principalmente no âmbito da APS ${ }^{18}$, enquanto os gastos estaduais são variados, e a participação dos gastos federais cresceu em termos de volume total de recursos, mas não em termos de participação relativa no conjunto do gasto em saúde, de participação no PIB ou na receita federal19,20.

O contexto nacional atual de reformas econômicas centradas na austeridade e em incentivos à expansão da participação privada em vários setores é desfavorável a mudanças radicais no financiamento das políticas públicas, o que torna imperativo amplo, responsável e democrático debate. O impacto social das transformações econômicas tende a gerar sobrecarga adicional para o SUS, em um cenário já marcado por instabilidade financeira e de precarização das relações de trabalho, em geral, e na saúde. A mudança apresentada não envolve aporte adicional de recursos federais para o SUS e rompe com as regras atuais do financiamento, havendo risco de se agravar a instabilidade para os municípios, dada a sua dependência das transferências intergovernamentais no financiamento de políticas de execução descentralizada, como a saúde.

O novo modelo de financiamento federal da APS traz alterações significativas e incertezas para a gestão municipal. O fim do PAB fixo compromete $\mathrm{o}$ aporte regular de recursos financeiros federais, importante fonte orçamentária para a totalidade dos municípios brasileiros. A implantação desse mecanismo de transferência nos anos 1990 favoreceu o fortalecimento da $\mathrm{AB}$ de forma acoplada ao processo de descentralização ${ }^{21}$, conferindo certa estabilidade ao financiamento municipal do SUS nos anos subsequentes, em que pesem os seus valores baixos, aquém do necessário, e o fato de os demais componentes do financiamento terem se expandido de forma mais expressiva ${ }^{\mathbf{1}}$.

O fim do PAB Variável (no componente vinculado à implantação de Equipes de Saúde da Família), que se constituiu em uma indução financeira, em boa parte, responsável pelo aumento da cobertura da ESF nas últimas décadas ${ }^{22}$, significa o fim da prioridade para esse modelo assistencial que tem apresentando consistentemente melhores resultados do que outras formas de prestação de AB. Traz também instabilidade para a gestão municipal na contratação de profissionais, comprometendo o planejamento orçamentário e a organização da atenção.

A nova forma de cálculo proposta - por capitação, considerando pessoas cadastradas ao invés de equipes cadastradas e responsáveis pela atenção a pessoas e grupos sociais em seus 
territórios - traz ameaças ao modelo de atenção da ESF de base comunitária e familiar, pois não favorece a implantação de ações voltadas para o território e para as populações, cruciais para a promoção da saúde e do controle de agravos e doenças, além da assistência individual.

Além disso, a exigência, a priori, de cadastro e lista de pacientes para a transferência intergovernamental de recursos pode gerar instabilidades para a gestão municipal e fere o princípio da universalidade do SUS. Nesse sentido, a comparação com o financiamento adotado no Reino Unido se mostra inadequada, onde a capitação por lista de pacientes é usada para remunerar os médicos generalistas, mas não para a alocação de recursos para regiões de saúde, que considera critérios combinados de necessidades de saúde e busca de equida$\mathrm{de}^{23}$. Portanto, não é adequado comparar um mecanismo de pagamento de profissionais de saúde com mecanismos para transferências intergovernamentais, especialmente em um país imenso, diverso e federativo como o Brasil.

A ponderação da proposta de capitação segundo critérios demográficos, de vulnerabilidade socioeconômica e de tipologia rural urbana dos municípios definida pelo Instituto Brasileiro de Geografia e Estatística (IBGE) ${ }^{\mathbf{2 4}}$, embora indique possibilidade de alocação de recursos para municípios com maiores necessidades, não contempla de modo satisfatório a diversidade de situações existentes e as especificidades regionais e, particularmente, as diferenças de acessibilidade das populações aos serviços de saúde. Além disso, a tipologia de municípios do IBGE utilizada para definir grupos de municípios e respectivas metas de cobertura por equipe não foi criada para esse propósito (medir acessibilidade aos serviços de saúde) e pode gerar distorções, dada a heterogeneidade inter e intramunicipal.

É verdade que o cadastramento da população na ESF é fundamental e não se pode ignorar a ocorrência de problemas de cadastro na APS brasileira. No entanto, é oportuno lembrar que aconteceu nos últimos anos uma transição dos sistemas de informação na $\mathrm{AB}$, com muitos municípios ainda com dificuldades na implementação do E-SUS $\mathrm{AB}$, e que uma das razões da substituição do Sistema de Informação da Atenção Básica (Siab) pelo atual sistema foi a necessidade de ter registros individualizados; que o Programa Mais Médicos ajudou a aumentar e a tornar a cobertura estimada mais próxima da real; que o número de pessoas por equipe no Brasil já é bem maior que em outros países com sistema universal; que além das ações clínicas (incluindo consultas), as ESF desenvolvem ações coletivas, incluindo apoio a outras políticas sociais (Programa Bolsa Família por exemplo); que a crise econômica tende a provocar aumento da demanda por cuidado na ESF; que a ESF foi um importante exemplo de superação do modelo de financiamento federal por procedimentos; que a cobertura oficial da ESF é próxima da cobertura estimada pela Pesquisa Nacional de Saúde, na qual também foi evidenciado o acesso maior à ESF de populações em situação de risco e vulnerabilidade ${ }^{25}$. Problemas de cadastro pedem, pois, adequada caracterização, compreensão contextualizada das suas causas bem como intervenções que as considerem efetivamente.

O potencial de cobertura de 4 mil pessoas cadastradas para cada Equipe de Saúde da Família prevista para um dos grupos de municípios é extremamente elevada e não encontra equivalência na organização e prestação desses serviços, mesmo em países mais homogêneos e de renda alta, que apresentam melhores condições socioeconômicas e de saúde ${ }^{26}$. Ressalte-se que a forma de cálculo do número máximo de equipes com financiamento federal para cada município, vigente até o momento dessa mudança, considerava a possibilidade de cobertura de 2 mil pessoas por equipe, por exemplo, em qualquer tipo de município, sendo possível para o gestor planejar equipes com diferentes populações adscritas, a depender da dinâmica territorial e singularidades dos grupos populacionais. No novo modelo, há risco de que as equipes operem com número 
de pessoas cadastradas mais elevado (para viabilizar maior repasse de recursos com menor custo de contratação de profissionais e de manutenção dos serviços), o que, na prática, pode significar menos equipes cobrindo mais pessoas.

A própria capitação, que poderia ser um dos critérios (não único) de medida da 'cobertura efetiva' das equipes ou de auxílio ao dimensionamento adequado de pessoas por equipe, é adotada como um dos eixos estruturantes do financiamento, e com parâmetros que aumentam a responsabilidade clínica de equipes em boa parte já sobrecarregadas pela demanda assistencial.

A avaliação de desempenho é uma dimensão importante para o sistema de saúde e precisa ser continuamente aprimorada. $\mathrm{O}$ SUS e, em particular, a AB já compreendem, desde os anos 1990, sistemas de informações e de indicadores de saúde voltados para o monitoramento e avaliação dos processos de atenção e resultados sanitários. Acrescentemse esforços de estudos avaliativos periódicos e iniciativas de maior envergadura como o Programa Nacional de Melhoria do Acesso e da Qualidade da Atenção Básica (PMAQ-AB), descontinuado sem consistente e amplo debate sobre efeitos ao longo de seus três ciclos.

Ressalta-se que o fim do PAB Fixo e o término do financiamento por equipes implantadas, aliado ao grande peso conferido à capitação e avaliação de desempenho como condição para a transferência de uma parte importante dos recursos federais, cujos instrumentos não estão devidamente explicitados na proposta, podem agravar a instabilidade e comprometer o planejamento pelos gestores municipais, que já enfrentam grandes desafios em virtude do subfinanciamento do SUS. Cabe assinalar que o componente de desempenho do PAB Variável, criado em 2011 com o PMAQ-AB, consistiu em novo recurso financeiro, acompanhado de aprimoramento e atualização do PAB Fixo e do PAB Variável relativo à implantação de equipes, além de investimentos expressivos em reforma, ampliação e construção de unidades básicas de saúde. Na proposta atual, ao contrário, o que se pode ver é centralmente um remanejamento interno de recursos e a substituição de componentes de financiamento.

Os demais componentes da proposta - incentivos a ações estratégicas - permanecem aparentemente iguais quanto a sua forma de cálculo, aferição e critérios de repasse para os municípios, e não se sabe ao certo se sofrerão mudanças e interrupções de fluxos, em um contexto de forte contingenciamento de recursos pelo governo federal. Mesmo assim, é possível observar que a proposta não comporta nenhuma indução e apoio à conformação de equipes multiprofissionais ao acabar com incentivos financeiros específicos para os Nasf, estratégia relevante para a configuração de um modelo de atenção mais abrangente e integral. As mudanças no financiamento, se associadas a outras proposições recentes do Ministério da Saúde, como a adoção de uma carteira de serviços e a criação da Adaps, que prevê a possibilidade de contratação de serviços privados, suscita preocupações sobre a perspectiva de expansão do setor privado no âmbito da APS ${ }^{27}$. Isso porque, em conjunto, as medidas podem favorecer a precificação de serviços passíveis de contratação de terceiros com base em capitação associada a pacote de procedimentos. $\mathrm{O}$ risco é o avanço da mercantilização em um espaço da atenção no SUS até então predominantemente estatal e não mercantil28, podendo reduzir ainda mais o poder dos gestores locais e ampliar sua dependência aos agentes privados. Além disso, é preciso avaliar o real impacto do novo mecanismo de transferência federal e suas implicações para a redistribuição de recursos e para a compensação de desigualdades nas condições de financiamento e de gasto em saúde dos municípios na $\mathrm{AB}$.

Cabe lembrar, ainda, que tal mudança estrutural no financiamento da APS, exigindo grande esforço de compreensão e ação pelos gestores e equipes de saúde, deverá coincidir com as eleições e com o encerramento de gestões municipais em 2020. 


\section{Considerações finais}

Por tratar-se de mudanças significativas e potencialmente desestruturantes da APS e do SUS, consideramos ser ainda premente a ampliação do debate, com prazos adequados para a apreciação das propostas, discussão e eventual formulação de alternativas, envolvendo diversidade de atores como convém ao espaço democrático do SUS. Além disso, faz-se necessário preservar os instrumentos que favoreçam a responsabilidade e autonomia do gestor municipal (como o PAB fixo), e a possibilidade de definição da distribuição, composição, população e área de abrangência das Equipes de Saúde da Família, segundo as especificidades das dinâmicas populacionais e sociais dentro de cada município.

Não menos importante, foi a peça publicitária veiculada pelo governo federal, logo após a publicação da portaria, indicando que 50 milhões de brasileiros mais vulneráveis encontravam-se 'esquecidos' e desassistidos pela ESF e que, a partir das mudanças propostas pelo novo financiamento, seriam de fato incluídos. Tal mensagem é bastante questionável se considerarmos que milhões de brasileiros saíram da pobreza justamente por meio de políticas inclusivas implementadas pelos governos anteriores, experiência reconhecida mundialmente. Ademais, o atual presidente, antes mesmo da posse, provocou a saída de 8 mil médicos cubanos participantes do PMM, que atuavam justamente nas áreas mais vulneráveis do País, gerando desassistência imediata de aproximadamente 30 milhões de brasileiros, apenas em parte compensada até o momento. $\mathrm{O}$ marketing desconsidera também o trabalho de milhares de trabalhadores e trabalhadoras das Equipes de Saúde da Família e de gestores que há mais de 25 anos lutam e constroem no cotidiano do SUS uma das experiências mais bem sucedidas de APS abrangente, de base territorial e comunitária. Por fim, 'cadastro' não pode ser considerado sinônimo de acesso e cuidado.

O fortalecimento do papel do Ministério da Saúde no financiamento adequado da APS, com revogação dos dispositivos que prejudicam o financiamento público da saúde, como a EC 95/2016, desvinculações e demais medidas de austeridade e restrição aos gastos sociais e renúncia fiscal, parece representar caminho mais assertivo para promoção do aumento real e substantivo do acesso aos serviços de saúde e do volume de recursos para a APS e para o SUS. É desse tipo de medida, articulada com estratégias efetivas para melhorar o acesso, a qualidade e a articulação com demais componentes das redes de atenção, que a APS do SUS precisa.

\section{Colaboradores}

Melo EA (0000-0001-5881-4849)*, Almeida PF (0000-0003-1676-3574)*, Lima LD (00000002-0640-8387)* e Giovanella L (00000002-6522-545X)* participaram da concepção, redação e aprovação final do artigo. 


\section{Referências}

1. Facchini LA, Piccini RX, Tomasi E, et al. Desempenho do PSF no Sul e no Nordeste do Brasil: avaliação institucional e epidemiológica da Atenção Básica à Saúde. Ciênc. Saúde Colet. [internet]. 2006 [acesso em 2018 ago 24]; 11(3):669-681. Disponível em: http://www. scielo.br/scielo.php?script=sci_arttext\&pid=S1413 $81232006000300015 \& \operatorname{lng}=\mathrm{em}$.

2. Macinko J. Evaluation of the impact of the Family Health Program on infant mortality in Brazil, 19902002. J Epidemiol Community Health [internet]. 2006 [acesso em 2019 set 24]; 60(1):13-9. Disponível em: https://www.ncbi.nlm.nih.gov/pubmed/16361449.

3. Macinko J, Souza MFM, Guanais FC, et al. Going to scale with community-based primary care: An analysis of the family health program and infant mortality in Brazil, 1999-2004. Soc Sci Med [internet]. 2007 [acesso em 2019 set 2018]; 65(10):2070-80. Disponível em: https://www.ncbi.nlm.nih.gov/pubmed/17689847.

4. Aquino R, Oliveira NF. Impact of the Family Health Program on Infant Mortality in Brazilian Municipalities. Am J Public Heal [internet]. 2009 [acesso em 2019 ago 24]; 99(1):87-93. Disponível em: https:// www.ncbi.nlm.nih.gov/pmc/articles/PMC2636620/.

5. Guanais FC, Macinko J. Primary Care and Avoidable Hospitalizations: Evidence from Brazil. J Ambul Care Manag [internet]. 2009 [acesso em 2019 set 7]; 32(2):115-22. Disponível em: https://www.ncbi.nlm. nih.gov/pubmed/19305223.

6. Facchini LA, Thumé E, Nunes BP, et al. Governance and Health System Performance: National and Municipal Challenges to the Brazilian Family Health Strategy. In: Reich MTK, editores. Governing Health Systems. Brookline: Lamprey \& Lee; 2015. p. 203-36.

7. Starfield B. Atenção Primária: equilíbrio entre necessidades de saúde, serviços e tecnologia. Brasília, DF: UNESCO; Ministério da Saúde; 2002.

8. Campos GWS, Gutiérrez AC, Guerrero AVP, et al. Reflexões sobre a Atenção Básica e a Estratégia de Saú- de da Família In: Campos GWS, Guerrero AVP, organizadores. Manual de Práticas de Atenção Básica. Saúde ampliada e compartilhada. São Paulo: Hucitec; 2008. p.132-153.

9. Almeida PF. Atención Primaria de Salud em um sistema universal: El caso de Brasil. In: Giovanella L organizadora. Atención Primaria de Salud em Suramerica. Rio de Janeiro: Isags: Unasur; 2015. p.155-192.

10. Ministério da Saúde. E-Gestor. Informação e gestão da Atenção Básica [internet]. [acesso em 2019 out 11]. Disponível em: https://egestorab.saude.gov.br/paginas/ acessoPublico/relatorios/relHistoricoCoberturaAB. xhtml;jsessionid=9CgF+IeB6OrdRvxWQSDbQ7VP.

11. Brasil. Ministério da Saúde, Departamento de Atenção Básica. Política Nacional de Atenção Básica. Brasília, DF: Ministério da Saúde; 2011.

12. Noronha JC, Lima LD, Machado CV. O Sistema Único de Saúde - SUS. In: Giovanella L, Escorel S, Lobato LVC, et al, organizadores. Políticas e Sistema de Saúde no Brasil. Rio de Janeiro: Editora Fiocruz; 2012. p. 365-393.

13. Brasil. Medida Provisória $\mathrm{n}^{\circ} 890$, de $1^{\circ}$ de agosto de 2019. Institui o Programa Médicos pelo Brasil, no âmbito da atenção primária à saúde no Sistema Único de Saúde, e autoriza o Poder Executivo federal a instituir serviço social autônomo denominado Agência para o Desenvolvimento da Atenção Primária à Saúde [internet]. Diário Oficial daUnião. 1 Ago 2019. [acesso em 2019 nov 11]. Disponível em: http://www. planalto.gov.br/ccivil_03/_ato2019-2022/2019/Mpv/ mpv890.htm.

14. Souto LRF, Noronha JC. Dois tempos, uma agenda permanente: saúde é democracia, democracia é saúde. Saúde debate 2019; 43(121):296-301.

15. Brasil. Portaria $n^{\circ} 2.979$, de 12 de novembro de 2019. Institui o Programa Previne Brasil, que estabelece novo modelo de financiamento de custeio da Atenção Primária à Saúde no âmbito do Sistema Único de 
Saúde, por meio da alteração da Portaria de Consolidação $\mathrm{n}^{\circ} 6 / \mathrm{GM} / \mathrm{MS}$, de 28 de setembro de 2017. [internet]. Diário Oficial da União. 13 Nov 2019. [acesso em 2019 nov 13]. Disponível em: http://www.in.gov. br/en/web/dou/-/portaria-n-2.979-de-12-de-novembro-de-2019-227652180.

16. Reis JG, Harzheim E, Nachif MCA, et al. Criação da Secretaria de Atenção Primária à Saúde e suas implicações para o SUS. Ciênc. Saúde Colet. 2019; 24(9):3457-3462.

17. Castro MC, Massuda A, Almeida G, et al. Brazil's unified health system: the first 30 years and prospects for the future. The Lancet. 2019; S0140-6736(19)31243-7.

18. Castro ALB, Machado CV, Lima LD. Financiamento da Atenção Primária à Saúde no Brasil. In: Mendonça MHM, Matta GC, Gondim R, et al., organizadores. Atenção Primária à Saúde no Brasil: conceitos, práticas e pesquisa. Rio de Janeiro: Fiocruz; 2018. p. 73-93.

19. Servo L, Piola SF, Paiva AB, et al. Financiamento e gasto público de saúde: histórico e tendências. In: Melamed C, Piola SF, organizadores. Políticas públicas e financiamento federal do Sistema Único de Saúde. Brasília, DF: IPEA; 2011. p. 85-108.

20. Machado CV, Lima LD, Andrade CLT. Federal funding of health policy in Brazil: trends and challenges. Cad Saúde Pública [internet]. 2014 [acesso em 2019 out 11]; 30(1):187-200. Disponível em: http://www. scielo.br/scielo.php?script=sci_arttext\&pid=S0102$-311 X 2014000100187 \& \operatorname{lng}=$ en\&nrm=iso.

21. Mendes A, Marques R. O financiamento da Atenção Básica e da Estratégia Saúde da Família no Sistema Único de Saúde. Saúde debate. 2014; 38(103):900-916.

22. Solla JJSP, Reis AAC, Soter APM, et al. Mudanças recentes no financiamento federal do Sistema Único de Saúde: atenção básica à saúde. Rev Bras Saude Mater Infant [internet]. 2007 [acesso em 2019 out 11]; 7(4):495-502. Disponível em: http://www.scielo.br/pdf/rbsmi/v7n4/a18v7n4.pdf.
23. Boyle S. United Kingdom (England). Health system review. Health systems in transition. 2011; 13(1):1-483.

24. Instituto Brasileiro de Geografia e Estatística. Classificação e caracterização dos espaços rurais e urbanos do Brasil: uma primeira aproximação/IBGE, Coordenação de Geografia. Rio de Janeiro: IBGE; 2017.

25. Malta DC, Santos MAS, Stopa SR, et al. A Cobertura da Estratégia de Saúde da Família (ESF) no Brasil, segundo a Pesquisa Nacional de Saúde, 2013. Ciênc. Saúde Colet. [internet]. 2016 [acesso em 2019 out 17]; 21(2): 327-338. Disponível em: http://www. scielo.br/scielo.php?script=sci_arttext\&pid=S1413$-81232016000200327 \& \operatorname{lng}=\mathrm{en}$.

26. Giovanella L. A atenção primária à saúde nos países da União Européia: configurações e reformas organizacionais na década de 1990. Cad. Saúde Pública [internet]. 2006 [acesso em 2019 out 19]; 22(5):951-963. Disponível em: http://www.scielo.br/scielo.php?script=sci_arttext \&pid=S0102$-311 X 2006000500008 \& \operatorname{lng}=e n$.

27. Rede de pesquisa em APS. ABRASCO. Contribuição dos pesquisadores da Rede APS ao debate sobre as recentes mudanças na política de atenção primária propostas pelo MS [internet]. 2019. [acesso em 2019 out 19]. Disponível em: https://www.abrasco.org.br/site/ eventos/congresso-brasileiro-de-ciencias-sociais-e-humanas-em-saude/contribuicao-dos-pesquisadores-da-rede-aps-ao-debate-sobre-as-recentes-mudancas-na-politica-de-atencao-primaria/43125/.

28. Viana ALd'A, Fausto MRC. Atenção Básica e Proteção Social: universalismo x focalismo e espaço não mercantil da assistência. In: Viana ALd'A, Elias PEM, Ibañez N, organizadores. Proteção Social - Dilemas e Desafios. São Paulo: Hucitec; 2005. p. 150-168.

Recebido em 19/10/2019

Aprovado em 14/11/2019

Conflito de interesses: inexistente

Suporte financeiro: não houve 